\title{
Development of the Molybdenum Millimeter Wave Target Tile for ECRH Antenna Alignment after the Evacuation in LHD*)
}

\author{
Hiroe IGAMI ${ }^{1)}$, Ryoma YANAI ${ }^{1)}$, Satoshi ITO ${ }^{1)}$, Yasuo YOSHIMURA ${ }^{1)}$, Shin KUBO ${ }^{1,2)}$, \\ Hiromi TAKAHASHI ${ }^{1,3)}$, Takashi SHIMOZUMA ${ }^{1)}$, Toru Ii TSUJIMURA ${ }^{1,3)}$, \\ Masaki NISHIURA ${ }^{1)}$ and Yoshinori MIZUNO ${ }^{1)}$ \\ ${ }^{1)}$ National Institute for Fusion Science, National Institutes of Natural Sciences, Toki 509-5292, Japan \\ ${ }^{2)}$ Nagoya University, Nagoya 464-8603, Japan \\ ${ }^{3}$ SOKENDAI, (Graduate University for Advanced Studies), Toki 506-5292, Japan
}

(Received 7 December 2020 / Accepted 7 February 2021)

\begin{abstract}
Alignment of the quasi-optical millimeter wave antenna for the electron cyclotron resonance heating (ECRH) can be required even after the evacuation of the vacuum vessel in fusion oriented plasma experimental devices. We developed a molybdenum millimeter wave target tile and installed it as one of the protection tiles of the vacuum vessel wall of the Large Helical Device (LHD) on the opposite side of the ECRH antennas. Inside the target tile, a sheathed thermocouple is mounted to measure the temperature increase during the millimeter wave injection toward this tile. In magnetic field configurations where neither the first nor the second electron cyclotron resonance (ECR) exists, the measured temperature increased sufficiently to be detected by the millimeter wave injection of approximately $100 \mathrm{~kW}$ for $0.1 \mathrm{~s}$ toward the target tile. Under the assumption that the distance between the target point that brings the largest temperature increase and the target point just above the thermocouple head gives the offset of the target point to be corrected, the offset of the antenna injection angle was confirmed with an accuracy of $0.4^{\circ}$ with changing the target point shot by shot.
\end{abstract}

(C) 2021 The Japan Society of Plasma Science and Nuclear Fusion Research

Keywords: ECRH, antenna alignment, millimeter wave target, thermal diffusion analysis

DOI: $10.1585 /$ pfr. 16.2405066

\section{Introduction}

The electron cyclotron resonance heating (ECRH) and current drive (ECCD) is widely used in magnetically confined experimental plasma devices for plasma initiation, sustainment, controlling the confinement characteristics, and suppression of the magneto-hydro-dynamic (MHD) oscillations. In fusion oriented experimental devices in which millimeter waves are used for ECRH, the power deposition region can be precisely controlled by quasi optical antennas. Therefore, the alignment of the quasi optical antenna is very important for ensuring the accuracy of the deposition region. In practice, the misalignment of the quasi optical antenna can occur by following possible reasons, such as the distortion of the transmission components, the mechanical troubles of the steering system of the quasi optical mirror, and other issues.

Previously we have confirmed the antenna alignment with injecting the high power millimeter wave toward the Kapton or PVC target plate brought into the vacuum vessel under atmospheric pressure during the maintenance period of LHD. With checking the point where the temperature increases on the target surface by infrared (IR) camera after the injection of the high power millimeter wave, the offset

author's e-mail: igami.hiroe@nifs.ac.jp

*) This article is based on the presentation at the 29th International Toki Conference on Plasma and Fusion Research (ITC29). of the target point can be confirmed. However, this method cannot be applied after the evacuation because these plastic materials cannot be left inside the vacuum vessel after the evacuation.

With use of a graphite tile installed in the vacuum vessel as a target plate of the high power millimeter wave injection, the temperature increase on the plate after the start of the injection can be measured by IR camera located outside the vacuum vessel via the $\mathrm{BaF} 2$ vacuum window. This method is useful for checking the antenna alignment and available even after the evacuation. In LHD, the graphite tiles are installed to protect the strike point of the helical divertor legs. When the graphite tiles covered the strike points of the open geometry, we injected high power millimeter waves from the ECRH antennas installed in the horizontal outer (O-) port toward the graphite tiles located on the opposite side to measure the temperature increase by IR camera. After the baffle-structured closed helical divertor (CHD) [1] was constructed instead of the open helical divertors in 2012, the high power millimeter wave injection toward the opposite graphite tiles became difficult. This is because as shown in Fig. 1, the graphite tiles of the baffle plate and the dome structure of the CHD do not face toward the ECRH antennas. Moreover, damage to the cooling channels and the components 


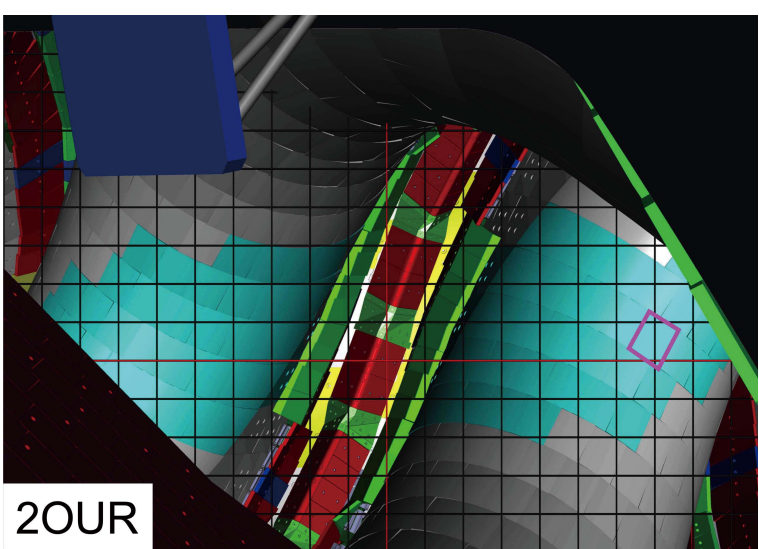

Fig. 1 The view from one of the ECRH antennas named UR installed in $2 \mathrm{O}$ port of LHD. The molybdenum protection tiles are indicated by aqua and the millimeter wave target tile is surrounded by magenta line. The baffle and dome plates of the CHD are indicated by green, blue, and red. The black grid interval corresponds to a $10 \mathrm{~cm}$ interval of the antenna target point on the virtual plane placed $3.9 \mathrm{~m}$ distant from the torus center along the toroidal direction at the center of the $2 \mathrm{O}$ port.

of the cryo-sorption pump installed inside the dome structure [2] caused by high power stray millimeter wave has been of more serious concern. We previously installed a round isotropic graphite target plate in a lower (L-) port that is located on the opposite side of an ECRH antenna installed in a U-port [3] to confirm the antenna alignment with using an IR camera. Before the start of the deuterium experiment on LHD, these IR cameras to monitor the temperature increase on the graphite targets located on the opposite side of the O-port and U-port ECRH antennas were removed. Because the cost of the equipment to protect the pixel of the IR cameras from the damage by neutrons and gamma rays is much expensive. Therefore we have had to develop a new target and a measurement system of the temperature increase on the target to confirm the antenna alignment after the evacuation.

In this paper, we first show the structure of the newly developed target tile and the measurement system of the temperature increase by millimeter wave injection in section 2. Predictions of the temperature increase at the measurement point with thermal diffusion analyses are presented in section 3 . The results of the temperature measurement with the high power millimeter wave injection toward the target tile are reported in section 4. Discussions and a summary are presented in section 5 .

\section{Structure of the Newly Developed Millimeter Wave Target Tile}

In LHD, molybdenum protection tiles are installed on the opposite side wall of the O-port ECRH antennas as shown in Fig. 1 to avoid the arc erosion when the injected millimeter wave reaches the opposite wall without

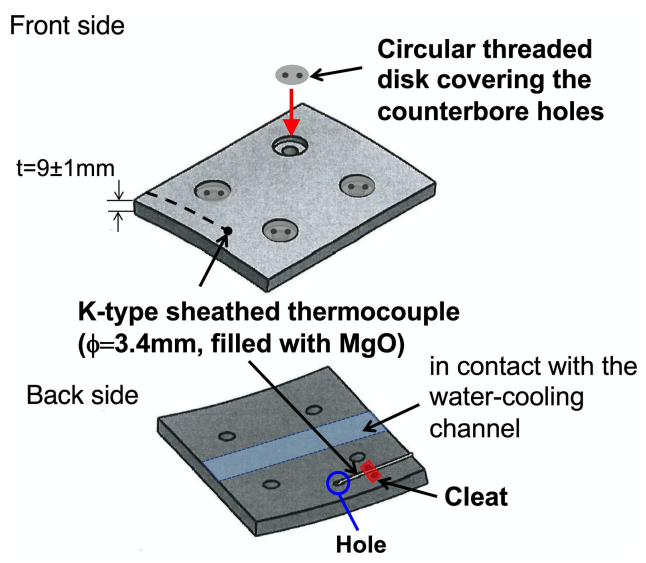

Fig. 2 Schematic structure of the molybdenum millimeter wave target tile.

the sufficient absorption inside the plasma. We replaced a molybdenum protection tile indicated by magenta line in Fig. 1 with a newly developed molybdenum target tile inside which the head of a sheathed K-type thermocouple whose diameter is $3.4 \mathrm{~mm}$ and filled with $\mathrm{MgO}$ is mounted at $2 \mathrm{~mm}$ depth from the front surface that faces to the ECRH antennas. Originally, this sheathed thermocouple was installed to monitor the temperature on the back surface of the protection tile and we have diverted the thermocouple to measure the temperature near the front surface. Figure 2 shows the schematic structure of the target tile. The counterbore holes of the front surface to fix the bolts are covered with molybdenum circular threaded disks to avoid the arcing at the edges of the holes during high power millimeter wave injection. A hole to insert the head of a Ktype sheathed thermocouple with bending by $45^{\circ}$ is drilled on the back surface. To fix the cleat that holds the sheathed thermocouple with two M3 bolts, two threaded holes are formed on the back surface. Because the sheathed thermocouple should be wired to avoid the central belt-like part that contacts the water cooling channel and also to avoid four fixing bolts, the head of the sheathed thermocouple is located distant from the center of the tile. The sheathed thermocouple line is extracted from the vacuum vessel at a lower port named $2.5 \mathrm{~L}$ via a vacuum flange and is connected via a compensating lead wire to a signal converter (MTT MS3701) inside a rack placed in the LHD experiment hall. The output of the signal converter is transmitted to the analog-digital converter (NI PXIe 4300) via a BNC cable to be acquired with the sampling frequency of $250 \mathrm{kHz}$.

\section{Estimation of the Temperature Increase with Thermal Diffusion Analyses}

To predict how much input energy on the target tile surface is required to measure the sufficient temperature increase to be detected, we performed thermal diffusion anal- 
yses by ANSYS $®$ and the one dimensional (1D) model. The absorption rate of the millimeter wave at the surface of the target tile is required to give the input power flux. In [4], it was reported that the measured and theoretically predicted ohmic losses which indicate the power absorption rates are $0.245 \pm 0.003 \%$ and $0.208 \%$ respectively for the perpendicular injection of the $140 \mathrm{GHz}$ millimeter wave to a molybdenum alloy TZM mirror. As suggested in [4], the actual absorption rate does not only depend on the material itself but also on the structure and the roughness of the surface. Moreover, the millimeter wave is not necessarily injected perpendicularly to the target tile. Nevertheless, we allow ourselves to assume that the millimeter wave is injected perpendicularly as a Gaussian beam to a flat molybdenum plate and is absorbed at the front surface with the absorption coefficient $a=0.25 \%$ for simplicity. The width, length, and thickness of the molybdenum plate are $120 \mathrm{~mm}, 144 \mathrm{~mm}$, and $9 \mathrm{~mm}$ respectively. Note that the actual shape of the target tile is curved as shown in Fig. 1, however, we consider a flat plate in this section.

For the description of the Gaussian beam intensity profile with the use of the cylindrical coordinate, we define $z$ direction along the beam axis and take $z$ as $z=z_{b}-z_{0}$. $z_{0}$ is the distance between the antenna and the focal point of the beam. $z_{b}$ is the distance from the antenna to the point on the beam axis where a line drawn from the concerned point along the radial direction meets. The length of this perpendicular line from the beam axis to the concerned point is given as $r$. The power flux intensity of the injected rounded Gaussian beam $p$, at the concerned point $(z, r)$ is given with taking into account the power of the millimeter wave $P_{\text {in }}$ as follows.

$$
\begin{aligned}
& p(z, r)=P_{i n} I(z, r)=\frac{2 P_{i n}}{\pi w_{b}^{2}} \exp \left[\frac{-2 r^{2}}{w_{b}^{2}}\right], \\
& w_{b}(z)=w_{0} \sqrt{1+\left(\frac{\lambda z}{\pi w_{0}^{2}}\right)^{2}} .
\end{aligned}
$$

Where $w_{b}$ is the beam radius that becomes $w_{0}$ at the focal point where $z=0$ and $w_{0}$ is the beam radius at the beam waist. $\lambda$ is the vacuum wave length, and $\pi$ is the circular constant. The values of the parameters $\lambda, w_{0}$, and $z_{0}$, and the values of the variables $w_{b}$, and $z_{b}$ to calculate the power flux used in the thermal diffusion analyses are indicated in Table 1. The value of $z_{b}$ was taken to be close to the distances from each antenna named UL, UR, LL, and LR to the point on the front surface of actual the target tile.

To denote the point on the flat molybdenum plate, here we introduce the Cartesian $\left(x^{\prime}, y^{\prime}, z^{\prime}\right)$ coordinate and place the origin at the center of the tetragon surrounded by corners on the front surface of the target plate where the input power flux is given by millimeter wave injection. For the analysis with the use of ANSYS $R$, the input power flux distribution on the flat molybdenum plate is given as $a p(z, r)$. Here $p(z, r)$ is given by equations (1) and (2) with the use of the values indicated in Table 1. And the ab-
Table 1 Parameters used to estimate the absorbed power on the target tile, when a rounded Gaussian beam is injected perpendicularly from each antenna named UL, UR, LL, and $\mathrm{LR}$ and installed in $2 \mathrm{O}$ port of LHD.

\begin{tabular}{|l|l|l|l|l|}
\hline & UL & UR & LL & LR \\
\hline$\lambda(\mathrm{mm})$ & 1.947 & 3.893 & 1.947 & 3.893 \\
\hline$W_{0}(\mathrm{~mm})$ & 35.0 & 65.0 & 38.1 & 35.0 \\
\hline$Z_{0}(\mathrm{~mm})$ & 2158 & 2965 & 2866 & 2920 \\
\hline$W_{b}(\mathrm{~mm})$ & 35.5 & 65.4 & 39.9 & 42.93 \\
\hline$Z_{b}(\mathrm{~mm})$ & 2876 & 3528 & 3843 & 3716 \\
\hline
\end{tabular}

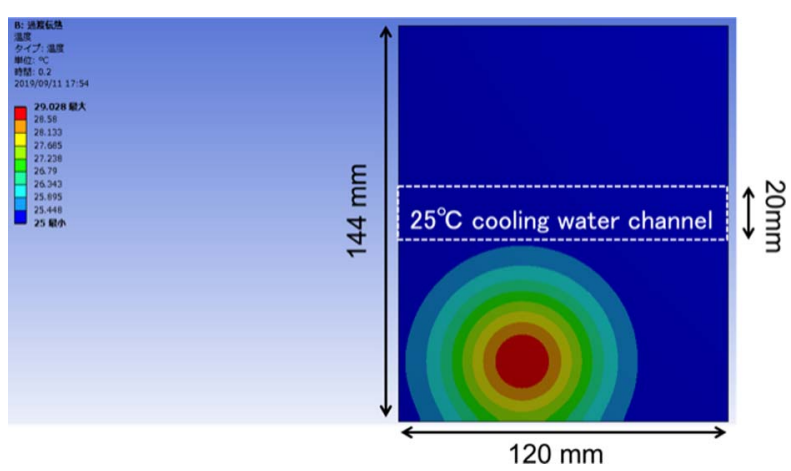

Fig. 3 Temperature distribution on the front surface of the flat target plate $200 \mathrm{~ms}$ after the start of $150 \mathrm{~kW}$ millimeter wave injection with rounded Gaussian beam profile. The assumed power absorption rate is $0.25 \%$. The Gaussian beam parameters $\left(\lambda, w_{0}, w_{b}, z_{0}, z_{b}\right)$ of LL antenna indicated in Table 1 are used.

sorption rate $a$ is taken to be $0.25 \%$. The values of the density, the specific heat capacity, and the heat conductivity are taken to be $10.22 \mathrm{~g} / \mathrm{cm}^{3}, 251 \mathrm{~J} / \mathrm{kg} / \mathrm{K}, 130 \mathrm{~W} / \mathrm{m} / \mathrm{K}$ respectively. As the boundary condition, the effect of the water cooling where the flat plate touches the cooling channel was taken into account with the use of the convective heat transfer coefficient $2000 \mathrm{~W} / \mathrm{m}^{2} / \mathrm{K}$. The power loss at the surfaces of the plate by radiation is not taken into account because they are negligible. For the 1D model analysis the power flux at the beam center is used to give the input power flux as $a p(z, r=0)$. Here $a$ is taken to be $0.25 \%$. The values of the density, the specific heat capacity, and the heat conductivity are taken to be $10.28 \mathrm{~g} / \mathrm{cm}^{3}$, $259 \mathrm{~J} / \mathrm{kg} / \mathrm{K}$, and $138 \mathrm{~W} / \mathrm{m} / \mathrm{K}$, respectively. The effect of the water cooling is not taken into account because the back surface of the target tile below the thermocouple head does not touch the cooling channel. In both thermal diffusion analyses, the uniform temperature $25^{\circ}$ Celsius is given as the initial condition.

Figure 3 shows the temperature distribution on the front surface $\left(z^{\prime}=0\right), 0.20 \mathrm{~s}$ after the start of the 150 $\mathrm{kW}$ millimeter wave injection for $0.2 \mathrm{~s}$ from LL antenna toward the target plate so that the beam center is located 

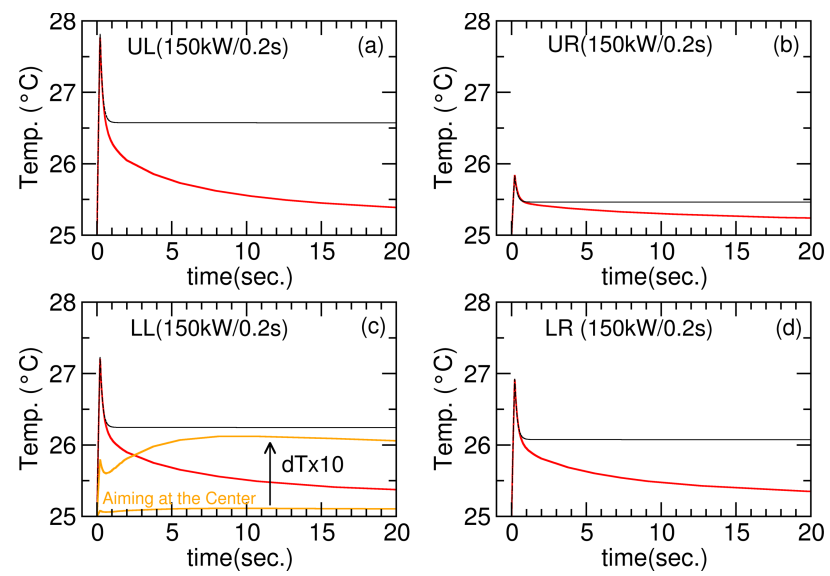

Fig. 4 Time evolutions of the temperature at the thermocouple head obtained by ANSYS $\AA$ (red lines) with assuming that the beam center is located just above the thermocouple and by the 1D model analysiswith assuming the input power flux at the beam center (black lines) plotted for each case of the millimeter wave injection from (a): UL, (b): UR, (c): LL, and (d): LR antennas. Time evolutions of the temperature $2 \mathrm{~mm}$ depth from the center of the frontsurface of the target plate obtained by ANSYS $\AA$ and its increment expanded by ten times (orange lines in panel (c)).

at the point just above the thermocouple where $\left(x^{\prime}, y^{\prime}\right.$, $\left.z^{\prime}\right)=(-15 \mathrm{~mm},-50 \mathrm{~mm}, 0 \mathrm{~mm})$. As the results obtained by ANSYS $®$ and the 1D model analysis, the time evolutions of the temperature at the head of the thermocouple where $\left(x^{\prime}, y^{\prime}, z^{\prime}\right)=(-15 \mathrm{~mm},-50 \mathrm{~mm},-2 \mathrm{~mm})$ are shown in panels of Fig. 4 for each case of $150 \mathrm{~kW} / 0.2 \mathrm{~s}$ millimeter wave injection from (a): UL, (b): UR, (c): LL, and (d): LR antennas, respectively. The target point of the injected beam was set on the front surface to be located just above the thermocouple for each case. Different from the time evolutions obtained by ANSYS $®$, the time evolutions obtained by the $1 \mathrm{D}$ model analysis remain almost constant after the rapid transient change during the millimeter wave injection because the effect of the water cooling is not taken into account. On the other hand, the time evolutions and the value at the maximum obtained by ANSYS $₫$ and the $1 \mathrm{D}$ model are almost the same during the rapid transient phase. The time evolution of the temperature at the point $2 \mathrm{~mm}$ distant from the center of the front surface of the target plate is obtained by ANSYS $R$ and also plotted for the similar case of the millimeter wave injection from LL antenna in the panel (c) of Fig. 4. Because of the horizontal thermal diffusion, the temperature increases after the stoppage of the millimeter wave injection. However, the increment cannot reach $0.1^{\circ}$ Celsius even at the maximum. Therefore, we can guess inversely that if the beam center is located at the center of the front surface of the target tile, we cannot observe a significant temperature increase to be detected at the thermocouple head since the sensitivity of the temperature measurement system is more than $0.1^{\circ}$ Celsius.
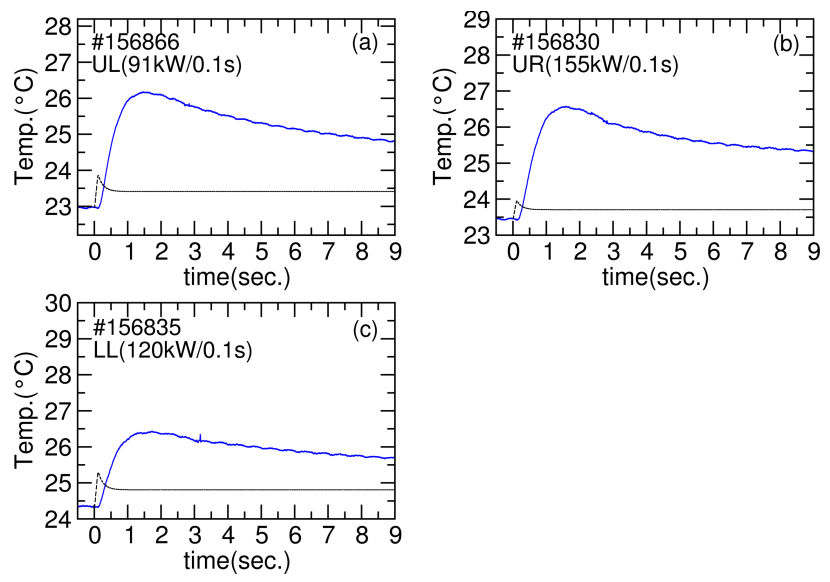

Fig. 5 Time evolutions of the measured temperature when the millimeter wave is injected for $0.1 \mathrm{~s}$ from (a): UL, (b): UR, (c): LL antennas with the injection power of $91 \mathrm{~kW}$, $155 \mathrm{~kW}$, and $120 \mathrm{~kW}$, respectively (blue lines) and time evolutions of the temperature obtained by the 1D model analysis with using the power flux at the beam center that is located on the target tile just above the thermocouple (black dash).

\section{Experimental Results}

After the evacuation of the vacuum vessel of LHD, we injected the millimeter wave for $0.10 \mathrm{~s}$ from each antenna installed in $2 \mathrm{O}$ port. Because we selected magnetic configurations where neither the first nor the second ECR exists inside the vacuum vessel, no plasma was started up by millimeter wave injection. Each power injected from UL, UR, and LL antennas was $91 \mathrm{~kW}, 155 \mathrm{~kW}$, and $120 \mathrm{~kW}$, respectively. Unfortunately, the injection from LR antenna could not be performed. For each antenna, we scanned the target point shot by shot to observe the largest temperature increase. Panels of Fig. 5 show the time traces of the measured temperature when the largest temperature increase was observed. We also show the time evolution of the temperature obtained by the $1 \mathrm{D}$ model analysis. In the 1D model analysis, the input power flux is taken to be the same as that at the center of the Gaussian beam injected in the experiment and the pulse length is also the same as that in the experiment. Differing from the time evolution obtained by the 1D model analysis, the measured temperature started to increase approximately $0.15 \mathrm{~s}$ after the start of the millimeter wave injection and reached the maximum about $1.4 \mathrm{~s}$ after the end of the power injection. The time constant of the sheathed thermocouple used in this study is approximately $1.4 \mathrm{~s}$. This time constant is not sufficient to detect the rapid time evolution predicted by the thermal diffusion analyses and causes the time delays of the measured temperature increase mentioned above. With taking into account the time delay, the temperature increase at the measured maximum $d T_{\max }$ from the temperature before the millimeter wave injection should be less than the temperature increase at the maximum obtained by $1 \mathrm{D}$ model analysis $d T_{p}$, however, as indicated in Table $2, d T_{\max }$ is larger 


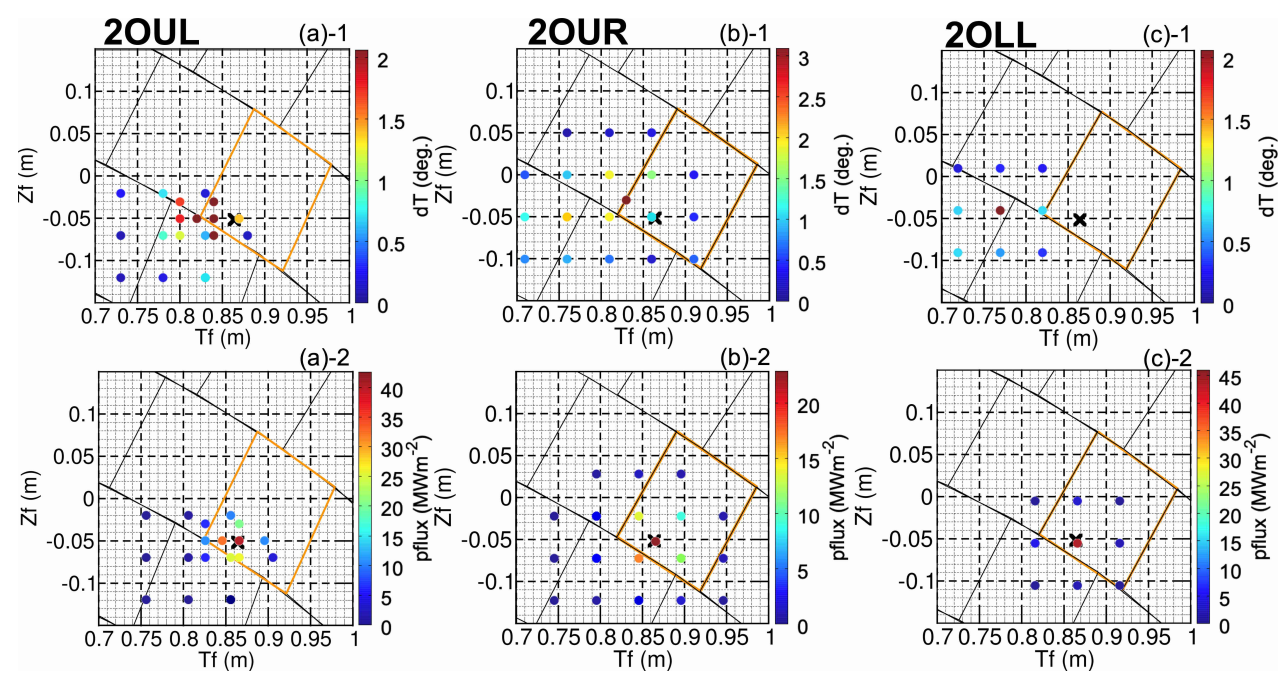

Fig. 6 Increase of the temperature at the measured peak from the temperature before the millimeter wave injection $(d T)$ and the power flux at the point on the target tile just above the thermocouple $(p f l u x)$ plotted versus the target point projected on the plane placed $3.13 \mathrm{~m}$ distant from the torus center along the toroidal direction at the center of the 20 port for injection cases of $91 \mathrm{~kW}$ from UL, $155 \mathrm{~kW}$ from UR and $120 \mathrm{~kW}$ from LL antennas. The target point just above the thermocouple is indicated by black cross mark. The maximum value of the color bar corresponds to the maximum of the measured temperature increase and the power flux at the beam center.

Table 2 Power flux at the beam center $\left(p_{c}\right)$, temperature increases from time $=0$, at the measured maximum $\left(d T_{\max }\right)$ and at the peak $\left(d T_{p}\right)$ and at the almost constant phase $\left(d T_{c}\right)$ calculated by $1 \mathrm{D}$ thermal diffusion analysis and the ratio of $d T_{\max }$ to $d T_{c}$, and $d T_{c}$.

\begin{tabular}{|l|l|l|l|}
\hline & UL & UR & LL \\
\hline$p_{c}\left(\mathrm{MW} / \mathrm{m}^{2}\right)$ & 42.54 & 22.89 & 45.97 \\
\hline$d T_{\max }($ deg. $)$ & 3.15 & 3.09 & 2.06 \\
\hline$d T_{p}$ (deg.) & 0.89 & 0.48 & 0.96 \\
\hline$d T_{\max } / d T_{p}$ & 3.54 & 6.43 & 2.15 \\
\hline
\end{tabular}

than $d T_{p}$ in any case of the injection from (a): UL, (b): UR, and (c): LL antennas. This fact suggests that the actual power absorption rate is larger than that used in the thermal diffusion analyses.

The distribution of $d T_{\max }$ plotted versus target points set in the experiment are shown in top panels (a)-1, (b)-1, (c)-1 and (d)-1 of Fig. 6 for injections from UL, UR, and LL antennas respectively. With scanning of the target point projected on the virtual plane that is placed $3.13 \mathrm{~m}$ distant from the torus center along the toroidal direction at the center of the $2 \mathrm{O}$ port by $5 \mathrm{~cm}$, we searched a target point that brings the largest temperature increase. With more precise scanning by $2 \mathrm{~cm}$ around the target point mentioned above, even larger increase was observed as shown in panel (c)-1 in Fig. 6 for the injection from UL antenna. Here we assume that the distance between the target point that brings the largest temperature increase and the target point just above the thermocouple gives the offset of the antenna target point to be corrected. The $2 \mathrm{~cm}$ offset of the target point on the virtual plane shown in panel (c)-1 of Fig. 6 corre- sponds to the $0.4^{\circ}$ offset of the injection angle.

In bottom panels (a)-2, (b)-2, (c)-2 and (d)-2 in Fig. 6, the distribution of the power flux at the point just above the thermocouple is plotted versus the target points set in the experiment to compare with the plots in the panels (a)-1, (b)-1, (c)-1 and (d)-1. Note that these target points in the bottom panels are corrected from those set in the experiment with taking into account of the offset. We find that the ratio of the measured temperature increase to the largest temperature increase tends to be larger than the ratio of the power flux to that at the beam center. This tendency might be caused by stray millimeter waves which come into the gaps between the neighboring protection tiles and are absorbed on the side surface and the back surface. Although these gaps do not exist in CAD data used in Fig. 1 and Fig. 6, actually approximately $5 \mathrm{~mm}$ gaps between protection tiles exist.

\section{Discussions and Summary}

We have developed a molybdenum millimeter wave target tile in which a thermocouple head is mounted to measure the temperature increase by injection of the high power millimeter wave. This target tile was installed on the opposite side wall of the ECRH antennas as one of the protection tiles of the vacuum vessel wall of LHD.

In magnetic configurations in which neither the first nor the second ECR exists, we injected the millimeter wave toward the target tile and observed the significant temperature increase by the thermocouple. Different from the thermal diffusion analyses with the use of ANSYS $®$ and 1D model, the measured temperature reaches the maximum $1.4 \mathrm{~s}$ behind after the end of the power injection, because 
the time constant of the sheathed thermocouple used in this study is approximately $1.4 \mathrm{~s}$. As the thermal diffusion analyses suggest, for the short pulse injection, the maximum temperature increase that appears in the rapid transient phase is proportional to the power flux at the point just above the thermocouple. It is necessary to measure the rapid transient time response of the temperature to detect the maximum correctly so that we can take the maximum temperature increase as the value proportional to the power flux at the point just above the thermocouple. To obtain the fast time response, we must use a sheathed thermocouple of smaller diameter. We found that the largest temperature increases obtained by the scanning of the target point are $2.15-6.43$ times larger than that predicted by heat diffusion analysis in the cases of the millimeter wave injection from UL, UR, and LL antennas. This fact suggests that the actual absorption rate of the millimeter wave might be larger than that which we used for the thermal diffusion analyses. Moreover, for estimating the absorption rate on the front surface of the target tile, we must eliminate the effect of the stray millimeter waves which penetrate into the gaps between the neighboring protection tiles and are absorbed on the side surface and the back surface. Blocking the stray millimeter waves with modification of the protection tiles, or developing some analytical technique to eliminate the effect of the stray millimeter wave absorption on the temperature increases is required. If we can measure the time evolution of the temperature with sufficiently fast time response and eliminate the effect of the stray millimeter wave absorption, we can obtain the beam profile on the target tile with precise scanning of the target point or installing a thermocouple array inside the target tile.

Although we could not interpret the maximum of the measured temperature increase as the value proportional to the power flux directly with the present measurement system as discussed above, we roughly estimated the offset of the target point with scanning the target point to observe the largest temperature increase. Under the assumption that the distance between the target point that brings the largest temperature increase and the target point just above the thermocouple head gives the offset of the target point to be corrected, the offset of the target point projected on the plane $3.13 \mathrm{~m}$ distant from the torus center is confirmed with an accuracy of $2 \mathrm{~cm}$ that corresponds to the offset of the injection angle of $0.4^{\circ}$.

In summary, we can roughly detect the offset of the antenna injection angle with changing the target point of the millimeter wave injected toward the molybdenum target tile to observe the largest temperature increase. Improving the time resolution of the temperature measurement and eliminating the effect of the stray millimeter waves are required to detect the maximum of the temperature increase during the transient phase correctly for the precise antenna alignment with obtaining the beam profile on the opposite side wall of the ECRH antennas.

\section{Acknowledgements}

The authors appreciate the technical staff for their support for this work. This work was carried out under Grant Nos. NIFS19ULRR701, NIFS19ULRR036 of the National Institute for Fusion Science, and partly supported by JSPS KAKENHI Grant Number 18K03590.

[1] T. Morisaki, S. Masuzaki, M. Kobayashi et al., Nucl. Fusion 53, 063014 (2013).

[2] T. Murase, G. Motojima, H. Tanaka et al., Plasma Fusion Res. 11, 1205030 (2016).

[3] H. Takahashi, S. Kubo, T. Shimozuma et al., EPJ Web of Conferences 87, 02019 (2015).

[4] W. Kasparek, A. Fernandez, F. Hollmann and R. Wacker, Int. J. Infrared Millim. Waves 22, 1695 (2001). 